\title{
Targeting Metabolism and Autophagy in the Context of Haematologic Malignancies
}

\author{
Versha Banerji ${ }^{1,2,3}$ and Spencer B. Gibson ${ }^{1,3,4}$ \\ ${ }^{1}$ Manitoba Institute of Cell Biology, 675 McDermot Avenue, Winnipeg, MB, Canada R3E 0V9 \\ ${ }^{2}$ Department of Internal Medicine, Faculty of Medicine, University of Manitoba, 770 Bannatyne Avenue, \\ Winnipeg, MB, Canada R3T 2N2 \\ ${ }^{3}$ CancerCare Manitoba, Section of Haematology/Oncology, 675 McDermot Avenue, Winnipeg, MB, Canada R3E OV9 \\ ${ }^{4}$ Biochemistry and Medical Genetics, Faculty of Medicine, University of Manitoba, 745 Bannatyne Avenue, \\ Winnipeg, MB, Canada R3E 0J9
}

Correspondence should be addressed to Spencer B. Gibson, gibsonsb@cc.umanitoba.ca

Received 13 April 2012; Accepted 28 May 2012

Academic Editor: Giuseppe Filomeni

Copyright ( 2012 V. Banerji and S. B. Gibson. This is an open access article distributed under the Creative Commons Attribution License, which permits unrestricted use, distribution, and reproduction in any medium, provided the original work is properly cited.

Autophagy is a cellular process that maintains the homeostasis of the normal cell. It not only allows for cell survival in times of metabolic stress with nutrient recycling but also is able to lead to cell death when required. During malignant transformation the cell is able to proliferate and survive. This is due to altered cell metabolism and the presence of altered genetic changes that maintain the cell survival. Metabolism was considered an innocent bystander that was a consequence of the increased nutrient requirement for the survival and proliferation of haematological malignancies. The interdependency of metabolism and cellular mechanisms such as autophagy are becoming more evident and important. This interdependence contributes to increased cancer progression and drug resistance. In this paper we aim to discuss autophagy, how it pertains to metabolism in the context of hematologic malignancies, and the implications for therapy.

\section{Introduction}

Autophagy was first described in the 1960s but its importance in various physiological conditions in addition to the basic molecular understanding of autophagy has only come into focus in the last decade. The word autophagy is derived from Greek: auto, meaning "self" and phagy, "to eat." This term was coined due to the process by which cellular components are degraded through the lysosomal enzymatic pathway providing a cell with essential amino acids, nucleotides, and fatty acids that enable production of the elements required for energy and macromolecule production $[1,2]$. Normal cells engage in autophagy as a means to survive disruptions in nutrient and growth factor availability. It also serves to eliminate damaged organelles and proteins to prevent accumulation. This prevents them from becoming toxic to the cell. If autophagy is prolonged to a point where normal cell function is compromised, cells undergo cell death either through apoptosis or by autophagy itself. One of the main inducers of autophagy is metabolic stress, and understanding the relationship between autophagy and metabolism could lead to better therapeutic strategies in treatment of haematological malignancies.

\section{Regulation of Autophagy}

Autophagy is characterized by cytoplasmic constituents sequestered into double-membraned vacuoles called autophagosomes. Autophagosomes then fuse with lysosomes (autolysosomes). Autolysosomes degrade cellular components releasing required nutrients to the cell. The regulation of autophagosome and autolysosome structures requires both positive and negative signaling pathways. The discovery in yeast of autophagy-related genes (ATGs) has provided greater understanding of these signaling pathways involved in autophagosome formation $[3,4]$. The initial signal to form 
autophagosomes is by the class III phosphatidylinositol (PI) 3 kinase complex consisting of Beclin1/Atg6, p150hVSp35, and class III PI3K (Vps34). This complex is required for formation of the preautophagosome structures [5]. Binding of ATG14, UVRAG (protein product of ultraviolet radiation resistant gene), and AMBRA1 (activating molecule of Beclin1-regulated autophagy) to the PI3K-III complex further increases the formation of autophagosomes allowing cells to regulate the amount of autophagy. AMBRA1 has also been shown to be a target of ULK1 [6]. ULK, TOR, FIP, Atg13, and AMPK represent molecules in the autophagy signaling network. The formation of the Beclin 1 complex is important to autophagosome formation. This process is negatively regulated by binding of Bcl-2 family members such as Bcl- $\mathrm{x}_{\mathrm{L}}$ to Beclin1 preventing Beclin1 binding to the PI3K-III complex and thereby reducing autophagy [5, 7].

Following PI3K-III complex induction of preautophagosome structures, a series of ATG proteins build autophagosomes using an ubiquitin-like mechanism. There are two ubiquitin-like mechanism used in autophagosome formation [8]. The first reaction is the ubiquitin-like protein ATG12 forming a conjugation to ATG5 via the E1 like protein Atg7 and E2-like protein ATG10 or ATG3. ATG16 then binds to the complex and integrates into the autophagosome membrane. The second reaction is the formation of the autophagosome membrane by ubiquitin-like protein LC3 (ATG8) conjugation with phosphatidyl ethanolamine (PE). This is regulated by ATG4 cysteine protease cleavage of LC3 at the C-terminus that facilitates lipidation of LC3 and generating LC3-PE conjugates. When both LC3-PE conjugates and Atg5-Atg12-Atg16 protein complex are localized to the autophagosome, the formation of autophagosomes is complete [8-10]. This process is regulated by acetylation of ATG-3 by histone acetyltransferase Esa- 1 suggesting that protein acetylation regulates autophagy [11].

Autophagosome formation is negatively regulated by the mammalian target of rapamycin (mTOR) pathway, a nutrient-sensing kinase pathway. Under growth conditions, the mTOR pathway regulates cell growth and survival but under nutrient starvation conditions, the mTOR pathway is inhibited allowing for induction of autophagy [5]. There are two different mTOR complexes: mTORC1 and mTORC2 [12]. mTORC1 complex contains $\mathrm{mTOR}$ and regulatory associated protein of mTOR (Raptor). The mTORC2 complex contains mTOR and rapamycin insensitive companion of mTOR (Rictor) [12, 13]. In nutrient rich conditions, the PI3K/AKT signaling pathway activates mTOR allowing formation of mTOR complexes and suppresses autophagy. This is through the mTORC1 where it binds to ULK1/2 (orthologue of yeast ATG1), mATG13, FIP200, and Atg101. Upon formation of this complex, mTOR phosphorylates ULK1 and ATG13 preventing ULK1 activation thereby blocking autophagosomes formation. In nutrient limiting conditions, the LBK/AMPK pathway is activated blocking mTOR activation. This is achieved by preventing mTORC1 binding to ULK complexes. This leads to ULK1 phosphorylation of ATG13 and FIP200 and autophagosome formation [12-14]. Alternatively, growth factor deprivation leads to activation of glycogen synthesis kinase-3 (GSK-3) that phosphorylates an acetyltransferase TIP60 which in-turn acetylates and activates ULK1 [15]. This leads to autophagy. Besides mTOR signaling, rubicon is also a negative regulator of autophagy and the normal maturation of the autophagosome. It thus serves as a brake in the autophagy process [16].

Autolysosomes are formed when autophagosomes and the lysosomes fuse. This allows the degradation of autophagosomal cargo. The lysosome proteins LAMP1 and LAMP2 are found in autolysosomes and are involved in degradation. In addition, the presence of cargo receptors or chaperone proteins, such as p62/SQSTM1 and NBR1, are responsible for sequestration of the ubiquitinated proteins into autophagosomes and autolysosomes [17]. Interactions between these autophagic adapters and the autophagosomal marker protein LC3, are required for efficient selective autophagy. The best characterized is p62/SQSTM1 that is responsible for delivering ubiquitinated proteins to autolysosomes for degradation [18]. Inefficient autophagic degradation of p62 leads to accumulation of ubiquitinated aggregates. This process is inhibited by reactivation of the mTOR pathway that causes the conversion of autolysosomes back to lysosomes. Thus, autophagy is a tightly regulated process that breaks down cellular constituents in response to cellular stress.

\section{Autophagy and Normal Haematopoiesis}

In the normal development of the erythrocyte, the reticulocyte is enucleated but retains its organelles. Through the process of autophagy, it then loses its organelles to become a circulating red blood cell [19]. Nix, also known as BNIP3L, has been demonstrated to play a role in this process through regulation of mitochondrial clearance [20]. Chemical differentiation of the $\mathrm{K} 562 \mathrm{CML}$ cell line also demonstrates a role for autophagy in megakaryocyte differentiation [21]. In lymphocytes autophagy proteins Atg5, Beclin1, and LC3 are upregulated in early thymocyte development and Tcell activation but downregulated in the mature CD4+ and CD8+ T cells [22]. Loss of autophagy gene Atg5 is important for B-cell survival during development. Loss of this gene leads to inefficient $B$ cell development characterized by increased cell death [23]. Autophagy is also known to regulate haematopoietic stem cells (HSCs) that are critical for normal hematopoiesis [24-26]. Recent studies showed that autophagic gene ATG7 is an essential regulator of adult HSCs maintenance since haematopoietic stem and progenitor cell lacking ATG7 expression have increased proliferation and DNA damage $[27,28]$. This confirms that autophagy is an important regulator of early development, homeostasis, and maintenance of haematopoiesis.

\section{Autophagy in Haematologic Malignancies}

The role of autophagy in haematologic malignancies is controversial [29, 30]. Autophagy has been shown to be either tumor promoting or tumor suppressive. Studies demonstrating a tumorigenic role of autophagy suggesting cancer cells can adapt and thrive to harsh environmental 
conditions such as low nutrients, growth factor deprivation, and metabolic stress because of autophagy [31-33]. This is due to the ability of autophagy to protect against apoptotic signaling through the degradation of damaged mitochondria, aggregated proteins, and pathogens within a cell $[30,34$, 35]. However, this distinct role of autophagy during tumor progression is cancer type specific depending on the developmental context and stage of cancer $[30,34,35]$. In normal haematopoeisis, autophagy regulates homeostasis. However, when this balance is disturbed the initiation of myelodysplastic syndrome (MDS) and acute myeloid leukemia (AML) ensues $[36,37]$. In addition, autophagy plays a role in cell survival in haematologic malignancies. This is illustrated by development of resistance to therapy such as chronic myelogenous leukemia resistance to imatinib [38].

Besides the role of autophagy in tumour progression, there is also evidence that supports a tumor suppressive role of autophagy. Beclin1, an autophagy gene, is found to be a haploinsufficient tumour suppressor in mice and is monoallelically deleted in human breast, ovarian, and other tumors but not in haematologic malignancies [39, 40]. p53 and PTEN are the most commonly mutated tumor suppressor genes and regulate autophagy in haematologic malignancies [41]. Through increased expression of autophagy genes such as DRAM, p53 increases autophagy where mutant p53 fails to increased autophagy gene expression and contributes to cell survival. Indeed, altered expression of autophagic genes Beclin1 or LC3 are considered prognostic markers in many tumours including non-Hodgkin lymphoma [42-44]. PTEN inhibits the PI3K/AKT pathway leading to decreased activation of the mTOR signaling pathway (Figure 1) and increased autophagy [45]. In contrast, mutant PTEN suppresses autophagy levels [46]. Autophagy can also function to promote apoptosis or induce cell death. Autophagic cell death has been demonstrated under various conditions in hematologic malignancies but the mechanisms that govern autophagy leading to tumour suppressive roles being unknown.

\section{Autophagy and Metabolism}

Autophagy is negatively regulated by growth factors, amino acids, and glucose signals leading to the nutrient responsive mTOR-signaling pathway [47] (Figure 1). Autophagy is regulated by adenosine monophosphate kinase (AMPK) via the mammalian target of rapamycin (mTORC1) pathway. AMPK senses changes in lipids and glucose to function as a metabolic sensor. It restores energy balance in the adenosine monophosphate (AMP) and adenosine triphosphate (ATP) ratio by the LKB1-AMPK activation. In AML the LKB1/AMPK pathway plays a tumor suppressor role through repression of mTOR-dependent mRNA translation [37]. Similarly, tumour necrosis inducing apoptosis ligand (TRAIL) is involved in apoptosis via intrinsic and extrinsic pathways. However, certain blood cancers such as chronic lymphocytic leukemia (CLL) are resistant to TRAIL-induced apoptosis. This could be due in part to TRAIL-induced cytoprotective autophagy. Thus, targeting autophagy genes such as Beclin 1 and Atg-5 enables TRAIL induced apoptosis $[48,49]$. Autophagy may play a role in the progression of low-risk MDS to AML by protecting the cells from extensive reactive oxygen species (ROS) induce damage from altered metabolism [37].

ROS play an important role in regulating metabolism and autophagy. ROS consist of unpaired electrons molecules such as superoxide $\left(\mathrm{O}_{2}^{-}\right)$, hydrogen peroxide $\left(\mathrm{H}_{2} \mathrm{O}_{2}\right)$, hydroxyl radical $\left(\mathrm{OH}^{-}\right)$, nitric oxide $\left({ }^{\bullet} \mathrm{NO}\right)$, peroxynitrite $\left(\mathrm{ONOO}^{-}\right)$, and nitrogen dioxide radical $\left({ }^{\bullet} \mathrm{NO}_{2}\right)$ [50-52]. Although ROS is formed from normal metabolism mainly from the mitochondria (Figure 1) and plays an important role in cell signaling and homeostasis leading to cell survival, ROS levels can increase causing irreversible oxidative damage leading to impaired metabolism and cell death [51, 53-55]. It has been documented that many chemotherapeutic agents raise levels of intracellular $\operatorname{ROS}[54,55]$. The essential role of mitochondria in generation of ROS and regulating tumorigenesis is implicated in many cancers including hematologic malignancies [36, 56]. Metabolic and oxidative stress also increases autophagy and blockage of ROS production or use of free radical scavengers inhibits autophagy. The mechanism of ROS induced autophagy is unclear but several possible mechanisms have been proposed. The cysteine protease Atg4 could be oxidized on a cysteine residue located near the active site, critical for its regulation. Atg4 regulates the reversible conjugation of Atg8 (LC3 in mammals) to the autophagosomal membrane, required for autophagosome formation [57]. Starvation-induced oxidative inactivation of ATG4 promotes lipidation of ATG8, facilitating autophagosome formation [57]. ROS accumulation could also be caused by selective autophagic degradation of catalase. Catalase degradation subsequently caused further ROS accumulation [58]. Other potential mechanisms for ROS regulation of autophagy could be through activation of transcription factor activity, leading to altered gene expression [59]. Indeed, autophagy genes are up-regulated in response to oxidative stress in yeast, and ROS induce Beclin1 and ATG-7 expression in different cancer cells. We have demonstrated that mitochondria are an important source of ROS leading to autophagy since oxidative phosphorylation inhibitors could induced autophagy mediated by ROS [51]. ROS induced autophagy has been shown to lead to cytoprotection and autophagic cell death. It was demonstrated that histone deacetylase inhibitor, SAHA induced autophagy and increased ROS leading to a cellular prosurvival mechanism in Jurkat Tcells [60]. In addition, FTY720, an immunosuppressive drug, induced cytoprotective autophagy in ALL [61]. In contrast, many chemotherapeutic drugs induce oxidative stress causing autophagic cell death. For example, increases in ROS, autophagosome formation and cell death have been detected upon Brevinin-2R treatment in Jurkat and BJAB (Bcell lymphoma) cells [62]. Another study showed that natural compound eupalinin-induced autophagic cell death through increased ROS in human leukemia cells [63, 64]. Taken together, increased cellular ROS production by therapeutic drug initiates a stress response leading to either cell survival or cell death. 


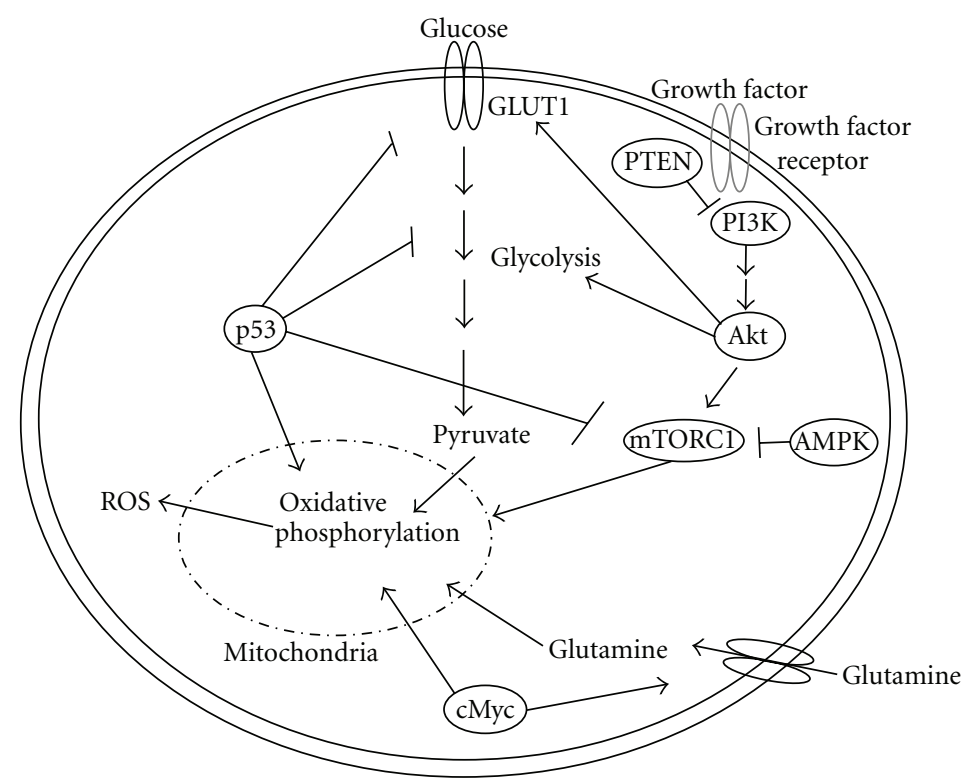

FIGURE 1: Metabolic signaling regulation. Glucose uptake in cells is regulated by Glut1 transporters that increase glycolysis and oxidative phosphorylation. This is enhanced by cMyc regulation of glutamine uptake in cells. Growth factors influence metabolism through activation of the PI3K/AKT/mTOR signalign pathway contribute to increased glycolysis and oxidative phosphorylation. This is inhibited through PTEN. Under glucose limiting conditions, AMPK is activated inhibiting the mTOR signaling pathway. In addition, p53 activaiton inhibits glycolysis, and the mTOR pathway but increases oxidative phosphorylation. ROS increases through inefficient oxidative phosphorylation at the mitochondria.

Both oncogene activation and tumor suppressor gene loss can all lead to deregulation of metabolic pathways such as glycolysis, pentose phosphate pathway, and lipid and energy metabolism. Cancer growth is dependent on functional mitochondria that are using glutamine as their major source of fuel for the citric acid cycle and the generation of NADPH and lipid synthesis [65]. The oncoprotein MYC activation is common in haematologic malignancies such as Burkitts lymphoma and AML. MYC upregulates glutamine transporters (Figure 1) and glutaminolysis, which increase ammonia production and autophagy protecting the cells from apoptosis $[66,67]$. $\mathrm{NF} \kappa \mathrm{B}$ activation is common in a variety of B-cell neoplasm including diffuse large B-cell lymphoma. Sommermann et al. showed that inhibition of $\mathrm{NF} \kappa \mathrm{B}$-induced cell death via the PI3K pathway and GLUT1 by restricting glucose transport [68]. To this end, it has been demonstrated that autophagy inhibitors in combination with NF $\kappa$ B induce a "metabolic crisis" and cell death [68]. Activating mutations in the oncogene Ras induce autophagy possibly through a novel AKT1-GLI3-VMP1 pathway [69]. This is essential for overcoming metabolic stress by impaired acetyl-CoA production leading to survival and tumor growth [70]. Overall, this demonstrates the interdependence of oncogene-mediated metabolic pathways and autophagy in response to cellular stress and cancer progression.

Besides oncogenes, tumour suppressors also regulate autophagy. Otto Warburg first observed that cancer cells undergo aerobic glycolysis due to lack of mitochondrial oxidative phosphorylation. The tumor suppressor, p53, positively regulates oxidative phosphorylation via synthesis of cytochrome c oxidase (COX-2) and downregulates glycolysis via transcription of TP53-induced glycolysis and apoptosis regulator (TIGAR) [71] (Figure 1). Loss of p53 enhances aerobic glycolysis resulting in more aggressive cancer phenotypes. p53 is often loss in cancer thus maybe an important genetic change contributing to the "Warburg effect." p53 is known as a regulator of apoptosis, however its role in coordination of nutrient utilization in order to preserve cell survival is equally important. TIGAR is a direct transcriptional target of p53 and alters cellular use of glucose. TIGAR shares sequence homology with the bisphosphatase domain of 6-phosphofructo2-kinase/fructose-2-,6-bisphosphataseand dephosphorylate fructose 2,6-bisphosphate reducing the levels of this metabolite. In addition, TIGAR suppresses ROS levels and autophagy. In the glycolytic pathway 6-Phosphofructo-1kinase (PFK-1) converts fructose 6 phosphate to fructose1, 6-bisphosphate. This in turn activates PFK-1 mediated by TIGAR and leads to inhibition of glycolysis. p53 also modulates another enzyme later in the glycolytic pathway, phosphoglyceratemutase (PGM). Wild type p53 downregulates PGM whereas p53 mutation increases its activity and leads glycolytic flux. In addition to regulation of glycolytic enzymes via TIGAR and PGM, p53 is important in the regulation of glucose transport. p53 can also down regulate glucose transporter expression leading to a reduction in intracellular glucose. Glucose transporter 1 (GLUT1) and glucose transporter 4 (GLUT4) are directly repressed at the gene promoter by p53 (Figure 1). This is important in autophagy since autophagy is activated by metabolic stress 
(glucose deficit) leading to degradation and recycling of cellular substrates that support metabolism and promote survival and tumor growth. p53 acts as a key regulator element autophagy through regulation of glycolytic pathway and hence metabolic stress.

The role of p53 in regulating autophagy through metabolism is complicated. It regulates through cellular location and by transcriptional dependent and independent mechanisms. Nuclear localization of p53 enables activation of AMPK which then leads to autophagy. A fine balance between nuclear and cytoplasmic p53 is responsible for autophagy homeostasis [72]. Nuclear p53 induces autophagy (Figure 2) through upregulation of mTOR pathway regulators. Under metabolic stress, basal p53 expression regulates multiple detoxifying pathways such as upregulation of antioxidant targets such as GPX1, MnSOD, ALDH4, and TPP53INP1 [73-77]. In addition, p53 target genes, sestrin1 and sestrin2, have been identified as a connection between p53 activation and mTORC1 activity [73, 78]. p53 exerts the antioxidant effect via inducing Sestrin expression in response to DNA damage and oxidative stress which leads to inhibition of mTORC1 activity and autophagy. Sestrins inhibit mTORC1 activity by interacting with MTOR pathway suppressors AMPK, TSC1, and TSC2 [78]. In contrast, cytoplasmic p53 inhibits autophagy mediated by activation of mTOR downstream signaling [79] (Figure 2). In addition, the mTOR pathway activates MDM2, the major ubiquitin ligase that reduces nuclear p53 expression [80]. Cytoplasmic p53 also binds to high mobility group box 1 (HMGB1) preventing formation the HMGB1/Beclin 1 complex, and inhibiting autophagy [81]. Beclin1 also controls the protein stabilities of ubiquitin-specific peptidases, USP10 and USP13, by regulating their deubiquitinating activities. Since USP10 mediates the deubiquitination of $\mathrm{p} 53$, regulating deubiquitination activity of USP10 and USP13 by Beclin1 provides a mechanism for Beclin1 to control the levels of p53 [82]. Moreover, p53 inhibition was found to promote cell survival in response to glucose starvation through autophagy [83]. All these results suggest that the autophagy induced by p53 deletion in tumors provide a survival advantage to malignant cells in response to unfavorable conditions. Taken together, p53 signaling regulates autophagy in response to metabolic stresses.

All these oncogenes and tumor suppressors play important roles in development and progression of hematological malignancies. Metabolic alterations are also a common feature in hematological malignancies. Thus, it is reasonable to suggest that these alterations regulated autophagy in hematological malignancy contributing to tumor survival and suppression. There are, however, many unanswered questions. What autophagy supplied substrates are essential for sustain metabolism? What affect do changes in metabolism and upstream signaling pathways have on autophagy in normal hematological stem cells or other immune cells? Nevertheless, targeting of autophagy regulatory pathways could provide treatments for hematological malignancies through either blocking or inducing autophagy.

\section{Targeting Autophagy and Metabolic Deregulation in Hematological Malignancies}

Chemotherapy or radiotherapy can both induce autophagy as a protective mechanism and lead to therapy resistance directly via mTOR inhibition and others indirectly by cytotoxic stress [84]. It may also cause chemoresistance by interfering with ROS activation that is the mechanism by which many chemotherapeutic agents function [84] (Figure 2). Inhibition of the proteasome induces autophagy and may pose reason for concern and resistance to therapy [85]. Hydroxychloroquine and chloroquine are known inhibitors of autophagy. They are also known antimalarials and thus clinically relevant compounds [86] (Figure 2). These agents have shown efficacy in targeting p53 loss induced autophagy and Myc induced autophagy in preclinical models $[87,88]$. Thus, the rational combination of an autophagy inhibitor chloroquine is being tested in clinical trial with bortezomib, a proteasome inhibitor, and cyclophosphamide in relapsed refractory multiple myeloma in a nonrandomized open label phase II clinical trial to determine if the combinatorial effects have clinical efficacy (http://clinicaltrials.gov/ct2/show/NCT01438177).

Many anticancer agents induce cell death through autophagy in hematologic malignancies instead of through cell survival by altering metabolism (Figure 2). For instance, arsenic trioxide $\left(\mathrm{As}_{2} \mathrm{O}_{3}\right)$ a potent antimetabolite exhibited potent antitumor effects through autophagic cell death in leukemic cell lines and primary leukemic progenitors from acute myelogenous leukemia (AML) patients [8991]. Moreover, arsenic trioxide-induced autophagy through inhibiting the mTOR pathway contributes to degradation of the PML/RARA fusion protein in acute promyelocytic leukemia (APL) [91, 92]. In addition, mTOR inhibitor NVP-BEZ235 treatment in T-ALL cells caused suppressing $\mathrm{PI} 3 \mathrm{~K} / \mathrm{Akt} / \mathrm{mTOR}$ signaling and induced autophagic cell death [93]. mTOR inhibitor RAD001 (Everolimus), also induced cell death by inducing autophagy in an in vivo model of childhood ALL [94, 95]. Resveratrol (RSV) is another attractive agent that induces autophagic cell death by inhibiting the AMPK/mTOR pathway in CML cells $[96,97]$ Histone deacetylase inhibitors are another class of agents that can be used to target autophagy. Although currently approved for the use of cutaneous T-cell lymphomas, suberoylanilide hydroxamic acid (SAHA) has been found to have activity in imatinib refractory CML. In addition, there is evidence to suggest that chloroquine maybe synergistic with SAHA in this clinical scenario $[98,99]$. Sphingolipids can also induce autophagy leading to increased apoptosis in leukemias and changes in sphingolipid metabolism have been observed in hematological malignancies $[100,101]$. Thus, targeting metabolic signaling pathways leading to autophagy could be an effective treatment of malignant hematologic disorders.

Finally, metformin, a biguanide, used to treat diabetes has been suggested as a potential anticancer drug. Metformin is a known LKB-1/AMPK activator (Figure 2). In melanoma, metformin was found to induce autophagy by increased expression of Beclin1, and accumulation of LC-3 secondary to mTOR inhibition leading to cell death [102]. Similar 


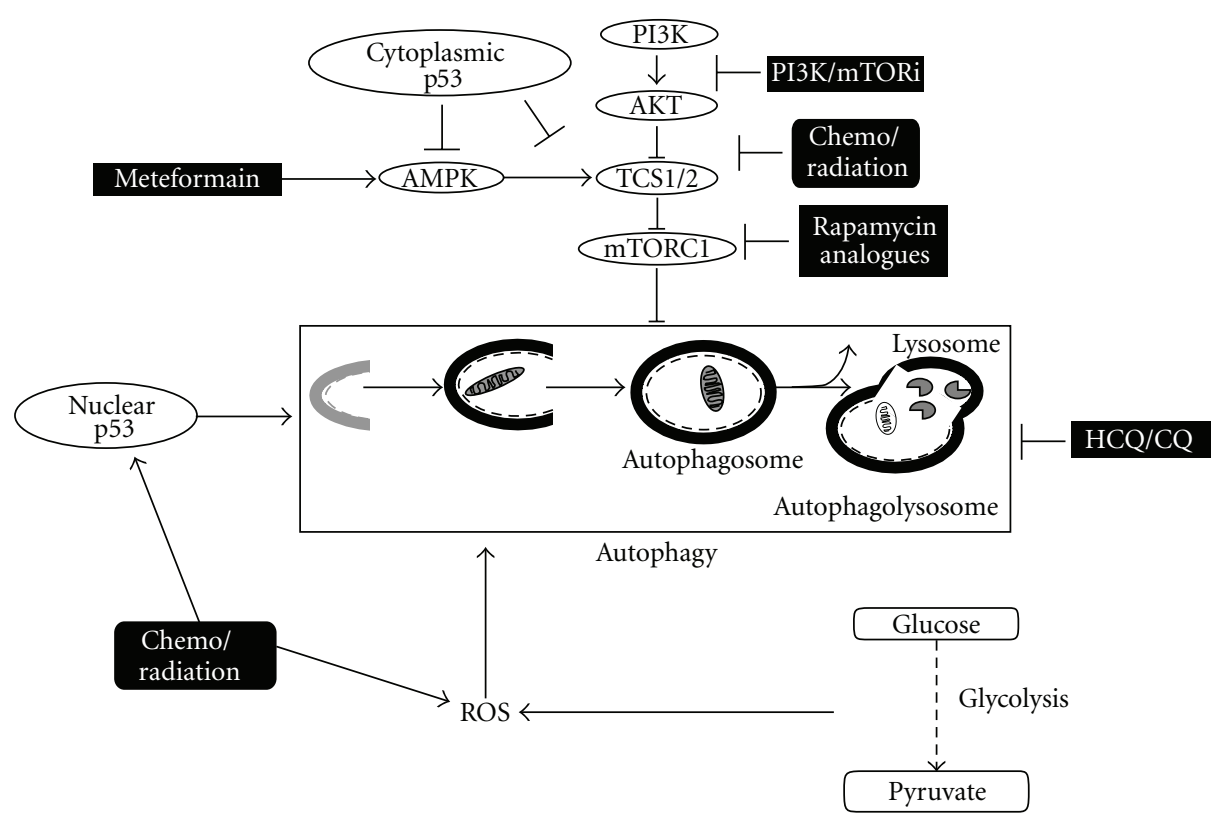

FIGURE 2: Signaling pathways regulating autophagy and their inhibitors. Autophagy is a catabolic process that results in the autophagosomiclysosomal degradation of bulk cytoplasmic contents. The kinase mTOR is a critical regulator of autophagy induction, with activated mTOR (PI3K/Akt) suppressing autophagy. AMPK-signaling negatively regulates mTOR signaling therefore promoting autophagy. ROS stress is an important inducer and regulator of autophagy generated by reduced oxidative phosphorylation and increased glycolysis. Nuclear 53 induces autophagy through transcriptional regulation of multiple genes whereas cytoplasmic p53 inhibits autophagy by blocking the mTOR pathway. Autophagy inhibitors chloroquine (HCQ/CQ) and metformin are under clinical investigation. Many chemotherapy/radiation therapies induce autophagy through ROS, inhibition of the mTOR pathway or nuclear p53 whereas PI3K/mTor inhibitors or rapamycin analogues specifically block the mTOR signaling pathway leading to autophagy.

effects have recently been described in lymphoma [103]. Metformin-induced activation of AMPK and inhibition of mTOR is AKT independent manner [103]. This lead to attenuated cell growth via induction of autophagy. The effect was evident in combination with doxorubicin versus single agent therapy and was reversed by autophagic inhibitor 3-methyladenine [103]. In T-ALL, metformin was found to have a significant antileukemic effect [104]. Metformin induced autophagy as evidenced by electron microscopy and increase in the LC3-II protein possibly contributing to cell death.

The major issue remains in hematological malignancies therapy as to whether induced or inhibited autophagy. The context of metabolism in cancer cells might be the key to this question and will govern the development of innovative metabolic therapies for hematological malignancies in the future.

\section{Conclusion}

The role of autophagy in cancer is multifaceted and its implication in metabolism is no different. This being said we are making headway in its understanding; however, there is more research required to understand the interactions between these currently distinct entities that are now merging in the pathogenesis of cancer. In hematologic malignancies it plays a role in pathogenesis, homeostasis, survival, and even cell death. An emerging role for metabolism has shed light on the interconnection between metabolism and autophagy. Metabolisms effect on autophagy is still ambiguous; it may lead to cell survival or cell death. Clinical evidence does support a role for metformin as an anticancer agent. It is also being looked at in the context of cancer prevention. In leukemia, it may be a realistic thought to use emerging technologies for metabolic profiling and treat patients in a personalized manner. The question that remains unanswered is whether to inhibit or activate autophagy as a treatment of hematological malignancies.

\section{References}

[1] D. Glick, S. Barth, and K. F. Macleod, "Autophagy: cellular and molecular mechanisms," Journal of Pathology, vol. 221, no. 1, pp. 3-12, 2010.

[2] E. A. Corcelle, P. Puustinen, and M. Jäättelä, "Apoptosis and autophagy: targeting autophagy signalling in cancer cells"trick or treats"?" FEBS Journal, vol. 276, no. 21, pp. 60846096, 2009.

[3] M. Thumm, "Isolation of autophagocytosis mutants of Saccharomyces cerevisiae," FEBS Letters, vol. 349, no. 2, pp. 275-280, 1994.

[4] M. Tsukada and Y. Ohsumi, "Isolation and characterization of autophagy-defective mutants of Saccharomyces cerevisiae," FEBS Letters, vol. 333, no. 1-2, pp. 169-174, 1993.

[5] M. Mehrpour, A. Esclatine, I. Beau, and P. Codogno, "Overview of macroautophagy regulation in mammalian cells,” Cell Research, vol. 20, no. 7, pp. 748-762, 2010. 
[6] S. Di Bartolomeo, M. Corazzari, F. Nazio et al., "The dynamic interaction of AMBRA1 with the dynein motor complex regulates mammalian autophagy," Journal of Cell Biology, vol. 191, no. 1, pp. 155-168, 2010.

[7] C. Liang, D. Sir, S. Lee, J. H. J. Ou, and J. U. Jung, "Beyond autophagy: the role of UVRAG in membrane trafficking," Autophagy, vol. 4, no. 6, pp. 817-820, 2008.

[8] I. Tanida, "Autophagy basics," Microbiology and Immunology, vol. 55, no. 1, pp. 1-11, 2011.

[9] N. Mizushima, "The role of the Atg1/ULK1 complex in autophagy regulation," Current Opinion in Cell Biology, vol. 22, no. 2, pp. 132-139, 2010.

[10] N. Mizushima and B. Levine, "Autophagy in mammalian development and differentiation," Nature Cell Biology, vol. 12, no. 9, pp. 823-830, 2010.

[11] C. Yi, M. Ma, L. Ran et al., "Function and molecular mechanism of acetylation in autophagy regulation," Science, vol. 336, no. 6080, pp. 474-477, 2012.

[12] S. Díaz-Troya, M. E. Pérez-Pérez, F. J. Florencio, and J. L. Crespo, "The role of TOR in autophagy regulation from yeast to plants and mammals," Autophagy, vol. 4, no. 7, pp. 851865, 2008.

[13] S. Y. Sun, "Enhancing perifosine's anticancer efficacy by preventing autophagy," Autophagy, vol. 6, no. 1, pp. 184-185, 2010.

[14] R. J. Shaw, "LKB1 and AMP-activated protein kinase control of mTOR signalling and growth," Acta Physiologica, vol. 196, no. 1, pp. 65-80, 2009.

[15] S.-Y. Lin, T. Y. Li, Q. Liu et al., "GSK3-TIP60-ULK1 signaling pathway links growth factor deprivation to autophagy," Science, vol. 336, no. 6080, pp. 477-481, 2012.

[16] H. Zhang, X. Kong, J. Kang et al., "Oxidative stress induces parallel autophagy and mitochondria dysfunction in human glioma U251 cells," Toxicological Sciences, vol. 110, no. 2, pp. 376-388, 2009.

[17] T. Johansen and T. Lamark, "Selective autophagy mediated by autophagic adapter proteins," Autophagy, vol. 7, no. 3, pp. 279-296, 2011.

[18] J. F. Dice, "Chaperone-mediated autophagy," Autophagy, vol. 3, no. 4, pp. 295-299, 2007.

[19] M. Kundu, T. Lindsten, C. Y. Yang et al., "Ulk1 plays a critical role in the autophagic clearance of mitochondria and ribosomes during reticulocyte maturation," Blood, vol. 112, no. 4, pp. 1493-1502, 2008.

[20] H. Sandoval, P. Thiagarajan, S. K. Dasgupta et al., "Essential role for Nix in autophagic maturation of erythroid cells," Nature, vol. 454, no. 7201, pp. 232-235, 2008.

[21] P. Colosetti, A. Puissant, G. Robert et al., "Autophagy is an important event for megakaryocytic differentiation of the chronic myelogenous leukemia K562 cell line," Autophagy, vol. 5, no. 8, pp. 1092-1098, 2009.

[22] H. H. Pua, I. Dzhagalov, M. Chuck, N. Mizushima, and Y. W. He, "A critical role for the autophagy gene Atg5 in T cell survival and proliferation," Journal of Experimental Medicine, vol. 204, no. 1, pp. 25-31, 2007.

[23] B. C. Miller, Z. Zhao, L. M. Stephenson et al., "The autophagy gene ATG5 plays an essential role in B lymphocyte development," Autophagy, vol. 4, no. 3, pp. 309-314, 2008.

[24] S. Bomken, K. Fišer, O. Heidenreich, and J. Vormoor, "Understanding the cancer stem cell," British Journal of Cancer, vol. 103, no. 4, pp. 439-445, 2010.

[25] J. E. Crowley, J. L. Scholz, W. J. Quinn III et al., "Homeostatic control of B lymphocyte subsets," Immunologic Research, vol. 42 , no. $1-3$, pp. 75-83, 2008.
[26] B. J. P. Huntly and D. G. Gilliland, "Leukaemia stem cells and the evolution of cancer-stem-cell research," Nature Reviews Cancer, vol. 5, no. 4, pp. 311-321, 2005.

[27] M. Mortensen, E. J. Soilleux, G. Djordjevic et al., "The autophagy protein Atg7 is essential for hematopoietic stem cell maintenance," Journal of Experimental Medicine, vol. 208, no. 3, pp. 455-467, 2011.

[28] M. Mortensen, A. S. Watson, and A. K. Simon, "Lack of autophagy in the hematopoietic system leads to loss of hematopoietic stem cell function and dysregulated myeloid proliferation," Autophagy, vol. 7, no. 9, pp. 1069-1070, 2011.

[29] M. M. Hippert, P. S. O’Toole, and A. Thorburn, "Autophagy in cancer: good, bad, or both?” Cancer Research, vol. 66, no. 19, pp. 9349-9351, 2006.

[30] S. Jin and E. White, "Role of autophagy in cancer: management of metabolic stress," Autophagy, vol. 3, no. 1, pp. 28-31, 2007.

[31] P. Boya, R. A. González-Polo, N. Casares et al., "Inhibition of macroautophagy triggers apoptosis," Molecular and Cellular Biology, vol. 25, no. 3, pp. 1025-1040, 2005.

[32] K. Degenhardt, R. Mathew, B. Beaudoin et al., "Autophagy promotes tumor cell survival and restricts necrosis, inflammation, and tumorigenesis," Cancer Cell, vol. 10, no. 1, pp. 51-64, 2006.

[33] J. J. Lum, D. E. Bauer, M. Kong et al., "Growth factor regulation of autophagy and cell survival in the absence of apoptosis," Cell, vol. 120, no. 2, pp. 237-248, 2005.

[34] M. N. Moore, J. I. Allen, and P. J. Somerfield, "Autophagy: role in surviving environmental stress," Marine Environmental Research, vol. 62, supplement 1, pp. S420-S425, 2006.

[35] B. Wolozin and C. Behl, "Mechanisms of neurodegenerative disorders. Part 1: protein aggregates," Archives of Neurology, vol. 57, no. 6, pp. 793-796, 2000.

[36] A. S. Watson, M. Mortensen, and A. K. Simon, "Autophagy in the pathogenesis of myelodysplastic syndrome and acute myeloid leukemia," Cell Cycle, vol. 10, no. 11, pp. 1719-1725, 2011.

[37] A. S. Green, N. Chapuis, T. T. Maciel et al., "The LKB1/AMPK signaling pathway has tumor suppressor activity in acute myeloid leukemia through the repression of mTOR-dependent oncogenic mRNA translation," Blood, vol. 116, no. 20, pp. 4262-4273, 2010.

[38] B. Calabretta and P. Salomoni, "Inhibition of autophagy: a new strategy to enhance sensitivity of chronic myeloid leukemia stem cells to tyrosine kinase inhibitors," Leukemia and Lymphoma, vol. 52, no. 1, pp. 54-59, 2011.

[39] X. H. Liang, S. Jackson, M. Seaman et al., "Induction of autophagy and inhibition of tumorigenesis by beclin 1," Nature, vol. 402, no. 6762, pp. 672-676, 1999.

[40] Z. Yue, S. Jin, C. Yang, A. J. Levine, and N. Heintz, "Beclin 1 , an autophagy gene essential for early embryonic development, is a haploinsufficient tumor suppressor," Proceedings of the National Academy of Sciences of the United States of America, vol. 100, no. 25, pp. 15077-15082, 2003.

[41] Z. Feng, H. Zhang, A. J. Levine, and S. Jin, "The coordinate regulation of the $\mathrm{p} 53$ and mTOR pathways in cells," Proceedings of the National Academy of Sciences of the United States of America, vol. 102, no. 23, pp. 8204-8209, 2005.

[42] Z. B. Ding, Y. H. Shi, J. Zhou et al., "Association of autophagy defect with a malignant phenotype and poor prognosis of hepatocellular carcinoma," Cancer Research, vol. 68, no. 22, pp. 9167-9175, 2008.

[43] S. Fujii, S. Mitsunaga, M. Yamazaki et al., "Autophagy is activated in pancreatic cancer cells and correlates with poor 
patient outcome," Cancer Science, vol. 99, no. 9, pp. 1813$1819,2008$.

[44] L. Pirtoli, G. Cevenini, P. Tini et al., "The prognostic role of Beclin 1 protein expression in high-grade gliomas," Autophagy, vol. 5, no. 7, pp. 930-936, 2009.

[45] S. Arico, A. Petiot, C. Bauvy et al., "The tumor suppressor PTEN positively regulates macroautophagy by inhibiting the phosphatidylinositol 3-kinase/protein kinase B pathway," The Journal of Biological Chemistry, vol. 276, no. 38, pp. 3524335246, 2001.

[46] D. J. Klionsky, H. Abeliovich, P. Agostinis et al., "Guidelines for the use and interpretation of assays for monitoring autophagy in higher eukaryotes," Autophagy, vol. 4, no. 2, pp. 151-175, 2008.

[47] M. Hoare, A. R. J. Young, and M. Narita, "Autophagy in cancer: having your cake and eating it," Seminars in Cancer Biology, vol. 21, no. 6, pp. 397-404, 2011.

[48] E. S. Henson, J. B. Johnston, and S. B. Gibson, "The role of TRAIL death receptors in the treatment of hematological malignancies," Leukemia and Lymphoma, vol. 49, no. 1, pp. 27-35, 2008.

[49] S. Fulda, "Cell death in hematological tumors," Apoptosis, vol. 14, no. 4, pp. 409-423, 2009.

[50] V. Adam-Vizi and C. Chinopoulos, "Bioenergetics and the formation of mitochondrial reactive oxygen species," Trends in Pharmacological Sciences, vol. 27, no. 12, pp. 639-645, 2006.

[51] Y. Chen and S. B. Gibson, "Is mitochondrial generation of reactive oxygen species a trigger for autophagy?" Autophagy, vol. 4, no. 2, pp. 246-248, 2008.

[52] J. D. Lambeth, "Nox enzymes, ROS, and chronic disease: an example of antagonistic pleiotropy," Free Radical Biology and Medicine, vol. 43, no. 3, pp. 332-347, 2007.

[53] J. P. Fruehauf and F. L. Meyskens, "Reactive oxygen species: a breath of life or death?" Clinical Cancer Research, vol. 13, no. 3, pp. 789-794, 2007.

[54] H. U. Simon, A. Haj-Yehia, and F. Levi-Schaffer, "Role of reactive oxygen species (ROS) in apoptosis induction," Apoptosis, vol. 5, no. 5, pp. 415-418, 2000.

[55] B. N. Lyu, S. B. Ismailov, B. Ismailov, and M. B. Lyu, "Mitochondrial concept of leukemogenesis: key role of oxygen-peroxide effects," Theoretical Biology and Medical Modelling, vol. 5, article 23, 2008.

[56] Y. Ohsumi, "Molecular dissection of autophagy: two ubiquitin-like systems," Nature Reviews Molecular Cell Biology, vol. 2, no. 3, pp. 211-216, 2001.

[57] R. Scherz-Shouval, E. Shvets, E. Fass, H. Shorer, L. Gil, and Z. Elazar, "Reactive oxygen species are essential for autophagy and specifically regulate the activity of Atg4," The EMBO Journal, vol. 26, no. 7, pp. 1749-1760, 2007.

[58] L. Yu, F. Wan, S. Dutta et al., "Autophagic programmed cell death by selective catalase degradation," Proceedings of the National Academy of Sciences of the United States of America, vol. 103, no. 13, pp. 4952-4957, 2006.

[59] G. Storz and B. S. Polla, "Transcriptional regulators of oxidative stress-inducible genes in prokaryotes and eukaryotes," EXS, vol. 77, pp. 239-254, 1996.

[60] J. Li, R. Liu, Y. Lei et al., "Proteomic analysis revealed association of aberrant ROS signaling with suberoylanilide hydroxamic acid-induced autophagy in jurkat T-leukemia cells," Autophagy, vol. 6, no. 6, pp. 711-724, 2010.

[61] C. T. Wallington-Beddoe, J. Hewson, K. F. Bradstock, and L. J. Bendall, "FTY720 produces caspase-independent cell death of acute lymphoblastic leukemia cells," Autophagy, vol. 7, no. 7, pp. 707-715, 2011.

[62] S. Ghavami, A. Asoodeh, T. Klonisch et al., "Brevinin-2R1 semi-selectively kills cancer cells by a distinct mechanism, which involves the lysosomal-mitochondrial death pathway," Journal of Cellular and Molecular Medicine, vol. 12, no. 3, pp. 1005-1022, 2008.

[63] T. Itoh, K. Ohguchi, Y. Nozawa, and Y. Akao, "Intracellular glutathione regulates sesquiterpene lactone-induced conversion of autophagy to apoptosis in human leukemia HL60 cells," Anticancer Research, vol. 29, no. 4, pp. 1449-1457, 2009.

[64] T. Itoh, Y. Ito, K. Ohguchi et al., "Eupalinin A isolated from Eupatorium chinense L. induces autophagocytosis in human leukemia HL60 cells," Bioorganic and Medicinal Chemistry, vol. 16, no. 2, pp. 721-731, 2008.

[65] F. Lozy and V. Karantza, "Autophagy and cancer cell metabolism," Seminars in Cell and Developmental Biology, vol. 23, no. 4, pp. 395-401, 2012.

[66] M. Yuneva, N. Zamboni, P. Oefner, R. Sachidanandam, and Y. Lazebnik, "Deficiency in glutamine but not glucose induces MYC-dependent apoptosis in human cells," Journal of Cell Biology, vol. 178, no. 1, pp. 93-105, 2007.

[67] C. H. Eng and R. T. Abraham, "Glutaminolysis yields a metabolic by-product that stimulates autophagy," Autophagy, vol. 6, no. 7, pp. 968-970, 2010.

[68] T. G. Sommermann, H. I. D. Mack, and E. Cahir-McFarland, "Autophagy prolongs survival after NF $\kappa$ B inhibition in B-cell lymphomas," Autophagy, vol. 8, no. 2, pp. 252-264, 2012.

[69] A. E. Lo Re, M. G. Fernandez-Barrena, L. L. Almada et al., "A novel AKT1-GLI3-VMP1 pathway mediates KRASinduced autophagy in cancer cells," The Journal of Biological Chemistry. In press.

[70] E. White, "Deconvoluting the context-dependent role for autophagy in cancer," Nature Reviews Cancer, vol. 12, no. 6, pp. 401-410, 2012.

[71] K. Bensaad, E. C. Cheung, and K. H. Vousden, "Modulation of intracellular ROS levels by TIGAR controls autophagy," The EMBO Journal, vol. 28, no. 19, pp. 3015-3026, 2009.

[72] E. Tasdemir, M. C. Maiuri, E. Morselli et al., "A dual role of p53 in the control of autophagy," Autophagy, vol. 4, no. 6, pp. 810-814, 2008.

[73] A. V. Budanov, J. H. Lee, and M. Karin, "Stressin' Sestrins take an aging fight," EMBO Molecular Medicine, vol. 2, no. 10, pp. 388-400, 2010.

[74] G. Pani and T. Galeotti, "Role of MnSOD and p66shc in mitochondrial response to p53," Antioxidants and Redox Signaling, vol. 15, no. 6, pp. 1715-1727, 2011.

[75] K. Bensaad, A. Tsuruta, M. A. Selak et al., "TIGAR, a p53inducible regulator of glycolysis and apoptosis," Cell, vol. 126, no. 1, pp. 107-120, 2006.

[76] W. Hu, C. Zhang, R. Wu, Y. Sun, A. Levine, and Z. Feng, "Glutaminase 2, a novel p53 target gene regulating energy metabolism and antioxidant function," Proceedings of the National Academy of Sciences of the United States of America, vol. 107, no. 16, pp. 7455-7460, 2010.

[77] S. Suzuki, T. Tanaka, M. V. Poyurovsky et al., "Phosphateactivated glutaminase (GLS2), a p53-inducible regulator of glutamine metabolism and reactive oxygen species," Proceedings of the National Academy of Sciences of the United States of America, vol. 107, no. 16, pp. 7461-7466, 2010.

[78] A. V. Budanov and M. Karin, "p53 target genes sestrin1 and sestrin 2 connect genotoxic stress and mTOR signaling," Cell, vol. 134 , no. 3 , pp. 451-460, 2008. 
[79] E. Tasdemir, M. C. Maiuri, L. Galluzzi et al., "Regulation of autophagy by cytoplasmic p53," Nature Cell Biology, vol. 10, no. 6, pp. 676-687, 2008.

[80] K. P. Lai, W. F. Leong, J. F. L. Chau et al., "S6K1 is a multifaceted regulator of $\mathrm{Mdm} 2$ that connects nutrient status and DNA damage response," The EMBO Journal, vol. 29, no. 17, pp. 2994-3006, 2010.

[81] K. M. Livesey, R. Kang, P. Vernon et al., "p53/HMGB1 complexes regulate autophagy and apoptosis," Cancer Research, vol. 72, no. 8, pp. 1996-2005, 2012.

[82] J. Liu, H. Xia, M. Kim et al., "Beclin1 controls the levels of p53 by regulating the deubiquitination activity of USP 10 and USP13," Cell, vol. 147, no. 1, pp. 223-234, 2011.

[83] M. C. Maiuri, L. Galluzzi, E. Morselli, O. Kepp, S. A. Malik, and G. Kroemer, "Autophagy regulation by p53," Current Opinion in Cell Biology, vol. 22, no. 2, pp. 181-185, 2010.

[84] R. Mathew and E. White, "Autophagy, stress, and cancer metabolism: what doesn't kill you makes you stronger," Cold Spring Harbor Symposia on Quantitative Biology, vol. 76, pp. 389-396, 2011.

[85] R. K. Amaravadi, J. Lippincott-Schwartz, X. M. Yin et al., "Principles and current strategies for targeting autophagy for cancer treatment," Clinical Cancer Research, vol. 17, no. 4, pp. 654-666, 2011.

[86] E. White and R. S. DiPaola, "The double-edged sword of autophagy modulation in cancer," Clinical Cancer Research, vol. 15, no. 17, pp. 5308-5316, 2009.

[87] R. K. Amaravadi, D. Yu, J. J. Lum et al., "Autophagy inhibition enhances therapy-induced apoptosis in a Myc-induced model of lymphoma," The Journal of Clinical Investigation, vol. 117, no. 2, pp. 326-336, 2007.

[88] K. H. Maclean, F. C. Dorsey, J. L. Cleveland, and M. B. Kastan, "Targeting lysosomal degradation induces p53dependent cell death and prevents cancer in mouse models of lymphomagenesis," The Journal of Clinical Investigation, vol. 118, no. 1, pp. 79-88, 2008.

[89] D. J. Goussetis, J. K. Altman, H. Glaser, J. L. McNeer, M. S. Tallman, and L. C. Platanias, "Autophagy is a critical mechanism for the induction of the antileukemic effects of arsenic trioxide," The Journal of Biological Chemistry, vol. 285, no. 39, pp. 29989-29997, 2010.

[90] W. Qian, J. Liu, J. Jin, W. Ni, and W. Xu, "Arsenic trioxide induces not only apoptosis but also autophagic cell death in leukemia cell lines via up-regulation of Beclin-1," Leukemia Research, vol. 31, no. 3, pp. 329-339, 2007.

[91] V. Charoensuk, W. P. Gati, M. Weinfeld, and X. C. Le, "Differential cytotoxic effects of arsenic compounds in human acute promyelocytic leukemia cells," Toxicology and Applied Pharmacology, vol. 239, no. 1, pp. 64-70, 2009.

[92] P. Isakson, M. Bjørås, S. O. Bøe, and A. Simonsen, "Autophagy contributes to therapy-induced degradation of the PML/RARA oncoprotein," Blood, vol. 116, no. 13, pp. 2324-2331, 2010.

[93] F. Chiarini, C. Grimaldi, F. Ricci et al., "Activity of the novel dual phosphatidylinositol 3-kinase/mammalian target of rapamycin inhibitor NVP-BEZ235 against T-cell acute lymphoblastic leukemia," Cancer Research, vol. 70, no. 20, pp. 8097-8107, 2010.

[94] R. Crazzolara, K. F. Bradstock, and L. J. Bendall, "RAD001 (everolimus) induces autophagy in acute lymphoblastic leukemia," Autophagy, vol. 5, no. 5, pp. 727-728, 2009.

[95] R. Crazzolara, A. Cisterne, M. Thien et al., "Potentiating effects of RAD001 (Everolimus) on vincristine therapy in childhood acute lymphoblastic leukemia," Blood, vol. 113, no. 14, pp. 3297-3306, 2009.

[96] A. Puissant and P. Auberger, "AMPK- and p62/SQSTM1dependent autophagy mediate resveratrol-induced cell death in chronic myelogenous leukemia," Autophagy, vol. 6, no. 5, pp. 655-657, 2010.

[97] A. Puissant, G. Robert, and P. Auberger, "Targeting autophagy to fight hematopoietic malignancies," Cell Cycle, vol. 9, no. 17, pp. 3470-3478, 2010.

[98] J. S. Carew, S. T. Nawrocki, F. J. Giles, and J. L. Cleveland, "Targeting autophagy: a novel anticancer strategy with therapeutic implications for imatinib resistance," Biologics, vol. 2, no. 2, pp. 201-204, 2008.

[99] J. S. Carew, S. T. Nawrocki, C. N. Kahue et al., “Targeting autophagy augments the anticancer activity of the histone deacetylase inhibitor SAHAto overcome Bcr-Abl-mediated drug resistance," Blood, vol. 110, no. 1, pp. 313-322, 2007.

[100] T. A. Burns and C. Luberto, "Sphingolipid metabolism and leukemia: a potential for novel therapeutic approaches," AntiCancer Agents in Medicinal Chemistry, vol. 11, no. 9, pp. 863881, 2011.

[101] D. Grandér, P. Kharaziha, E. Laane, K. Pokrovskaja, and T. Panaretakis, "Autophagy as the main means of cytotoxicity by glucocorticoids in hematological malignancies," Autophagy, vol. 5, no. 8, pp. 1198-1200, 2009.

[102] T. Tomic, T. Botton, M. Cerezo et al., "Metformin inhibits melanoma development through autophagy and apoptosis mechanisms," Cell Death and Disease, vol. 2, no. 9, article e199, 2011.

[103] W.-Y. Shi, D. Xiao, L. Wang et al., "Therapeutic metformin/AMPK activation blocked lymphoma cell growth via inhibition of mTOR pathway and induction of autophagy," Cell Death and Disease, vol. 3, no. 3, article e275, 2012.

[104] C. Grimaldi, F. Chiarini, G. Tabellini et al., "AMP-dependent kinase/mammalian target of rapamycin complex 1 signaling in T-cell acute lymphoblastic leukemia: therapeutic implications," Leukemia, vol. 26, no. 1, pp. 91-100, 2012. 

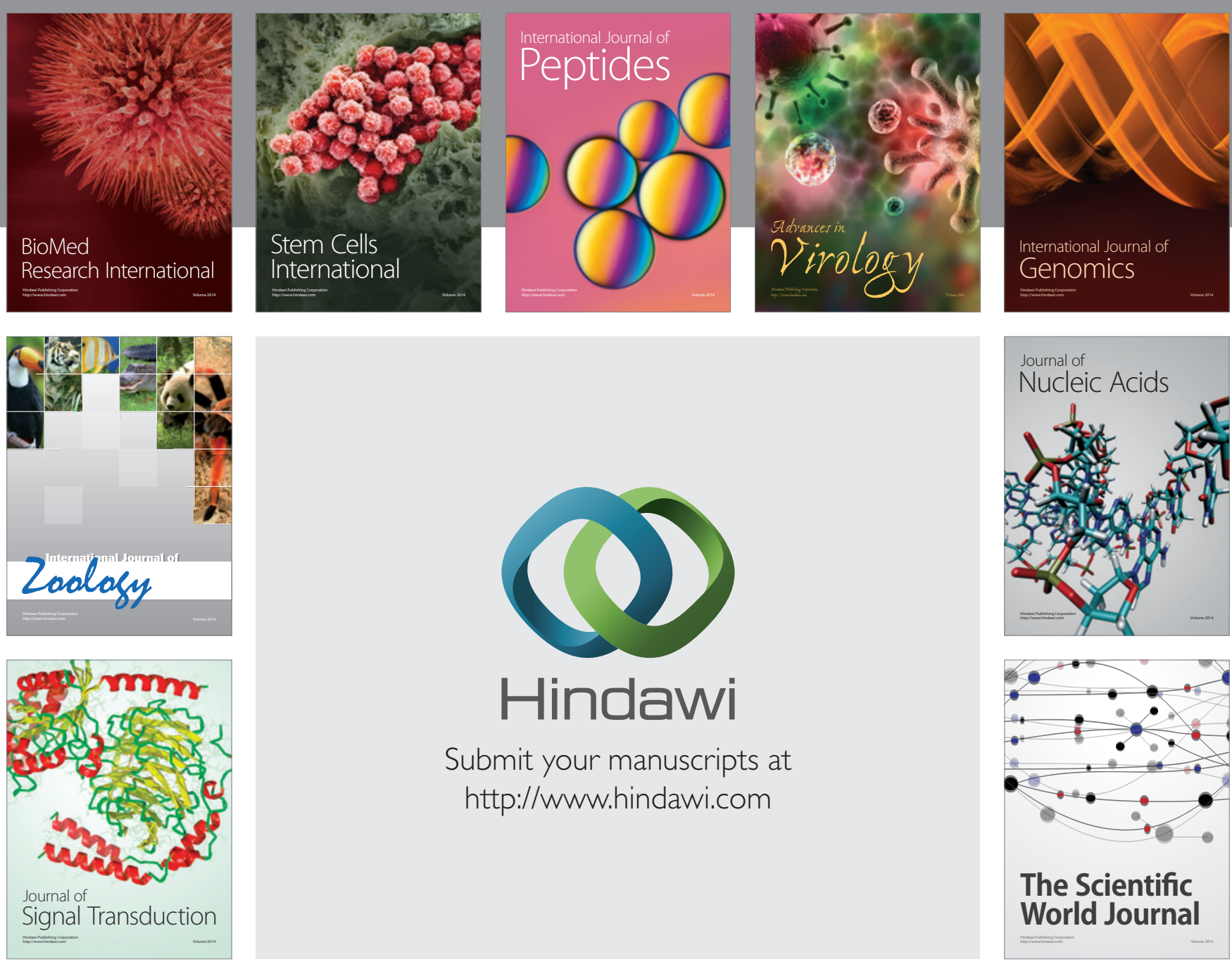

Submit your manuscripts at

http://www.hindawi.com
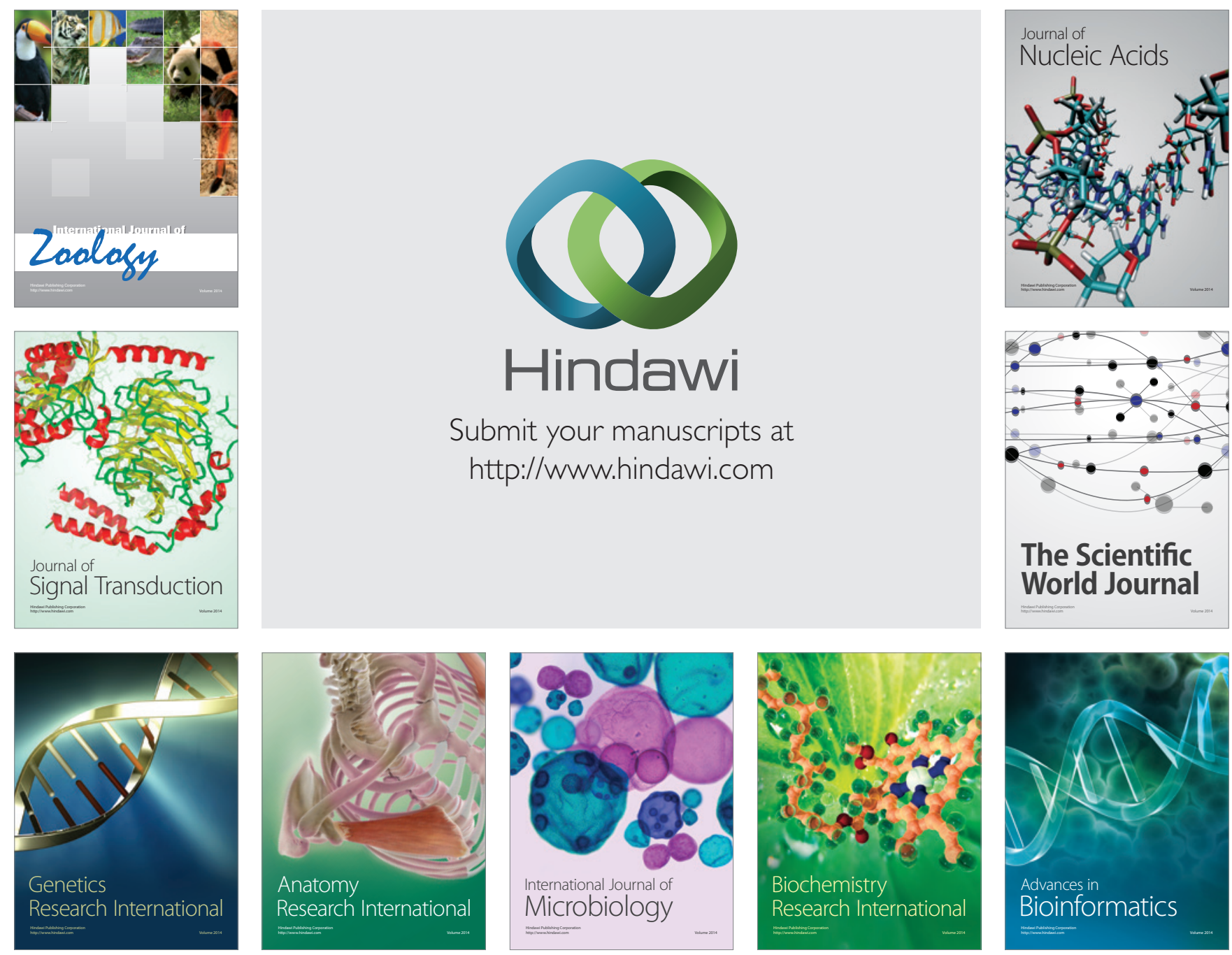

The Scientific World Journal
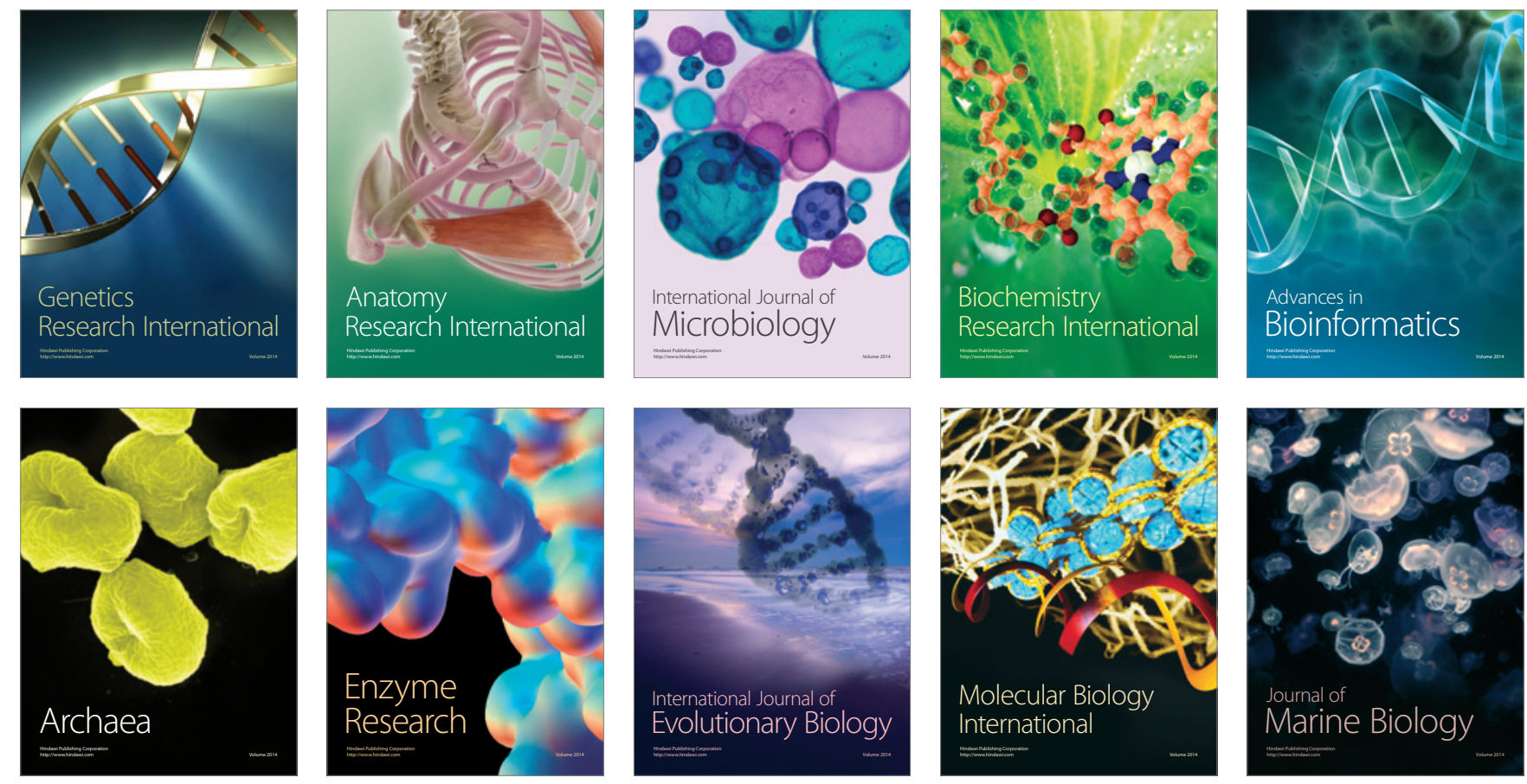